\title{
Asymmetric Autocorrelation Function To Resolve Directional Ambiguity In PIV Images
}

\author{
Y. M. Marzouk and D. P. Hart \\ Massachusetts Institute of Technology \\ Department of Mechanical Engineering \\ Cambridge, MA 02139-4307
}

\begin{abstract}
Autocorrelation of a double-exposed image, unlike cross-correlation between two images, produces a correlation function that is symmetric about the origin. Thus, while it is possible to calculate the speed and direction of tracer particles in a particle image velocimetry (PIV) image using autocorrelation, it is impossible to tell whether the velocity is in the positive or negative direction. This ambiguity can be resolved by spatially shifting one exposure relative to the next or labeling exposures with color or polarization, but the complexity and limitations of these methods can be prohibitive. It is, however, possible to resolve the sign of the velocity from a triple-exposed image using unequal time intervals between exposures.

Triple-exposed images, like double-exposed images, correlate symmetrically about zero. The directional ambiguity, however, can be resolved by calculating the probability that the three exposures occur in a specific temporal order; that is, by assuming that the correlation has a specific sign and testing to see if the assumption is correct. Traditional spectral and statistical correlation techniques are unable to accomplish this. Presented herein is a computationally efficient asymmetric correlation function that is able to differentiate the temporal order of triple exposed images. Included is a discussion of the limitations of this function and of difficulties in experimental implementation.
\end{abstract}

\section{NOMENCLATURE}

$\begin{array}{ll}\eta & \text { Normalized asymmetric correlation function } \\ \Phi & \text { Normalized correlation function } \\ \Phi^{o} & \text { Masked correlation function } \\ \phi^{e} & \text { Non-normalized error correlation function [pixels] } \\ \boldsymbol{\phi}^{s} & \left.\text { Non-normalized statistical correlation function [pixels }{ }^{2}\right] \\ \boldsymbol{\Delta} & \text { Correlation search length [pixels] } \\ \tau & \text { Asymmetric exposure ratio } \\ \boldsymbol{I} & \text { Pixel intensity } \\ \boldsymbol{i}, \boldsymbol{j} & \text { Image coordinates [pixels] } \\ \boldsymbol{m , n} & \text { Data array indices } \\ \boldsymbol{M}, \boldsymbol{N} & \text { Interrogation image dimensions [pixels] } \\ \boldsymbol{x}, \boldsymbol{y} & \text { Pixel image coordinates [pixels] } \\ \boldsymbol{\Delta i}, \boldsymbol{i} \boldsymbol{j} & \text { Pixel displacement [pixels] }\end{array}$




\section{INTRODUCTION}

Particle image velocimetry (PIV) has found wide application as a means of simultaneously and non-invasively measuring velocities in a plane within a flow field [Adrian 1991]. Typically, PIV proceeds by either cross-correlation or autocorrelation. For cross-correlation, a single exposure of tracer particles in the flow is recorded on two images taken at different times, while for autocorrelation, multiple exposures are recorded in a single image. Crosscorrelation of PIV images is in many cases not practical for high-speed flows; the time interval between video frames or the time necessary to advance film is long enough to lose any correlation of particle positions [Willert and Gharib 1991]. Autocorrelation, on the other hand, permits exposures to be as closely spaced as the light source (often a pulsed laser) will allow, provided that particle images do not overlap.

Particle images resulting from different exposures are indistinguishable in an image designed for autocorrelation; it is impossible to know the order in which individual particle images were recorded. Autocorrelation thus retains a $180^{\circ}$ ambiguity in the direction of each vector in the velocity field. For applications with vortex structures, velocity reversals, or strong turbulence, flow directions cannot be readily inferred, and it is important that directional ambiguity be resolved.

A variety of techniques has been proposed for this purpose. Some concentrate on labeling particle images from different exposures so they can be addressed independently and crosscorrelated. Labeling methods include illumination with distinct colors [Reeves et al. 1994], as well as polarization-dependent fringe encoding [Reeves et al. 1995]. Grant and Liu [1990], on the other hand, have developed a "pulse tagging" approach relying on two closely-spaced laser pulses of differing length. Image processing algorithms match pulse pairs to give flow directionality.

A more widespread technique, proposed by Adrian [1986], introduces a velocity bias by shifting the image of the second exposure with respect to the first. The length of the shift is chosen so that no negative particle displacements appear in the image. Velocity bias can be produced mechanically, by viewing the interrogation region with a mirror rotating at constant angular velocity [Landreth et al. 1988]. It can also be produced electrooptically, using perpendicular polarization for each laser pulse and viewing the interrogation region through a birefringent crystal or a system of waveplates [Landreth and Adrian 1988; Lourenco 1993].

All these techniques for resolving directionality add experimental complexity to the PIV system and increase the complexity of data analysis. Mechanical velocity bias techniques may introduce systematic errors in velocity measurements [Zhang and Eisele 1995; Oschwald et al. 1995], and all image shifting techniques come with limitations on the strength of the velocity bias they can impose. A simpler and more flexible system is desirable.

A new algorithm for resolving directional ambiguity is proposed, relying on three laser pulses per image, separated by unequal time intervals. Included is a discussion of the limitations of this algorithm and the difficulties in experimental implementation. 


\section{DIRECTIONAL AMBIGUITY}

PIV images are typically processed by subdivision into a regular grid of overlapping windows that bound regions of similar flow velocity; a velocity vector is then found for each window by autocorrelation. Autocorrelation produces a table of correlation values over a range of displacements, and the overall displacement of particles in the window is represented by a peak in this correlation table. Entries of the correlation table are typically computed using either spectral (FFT) autocorrelation or statistical autocorrelation. For an $\mathrm{M}$ by $\mathrm{N}$ window, the statistical autocorrelation function can be expressed as

$$
\Phi_{\Delta i, \Delta j}^{s}=\frac{\sum_{m=1}^{M} \sum_{n=1}^{N}\left[I_{m+\Delta i, n+\Delta j} \cdot I_{m, n}\right]}{\sqrt{\sum_{m=1}^{M} \sum_{n=1}^{N} I_{m, n}^{2}} \cdot \sqrt{\sum_{m=1}^{M} \sum_{n=1}^{N} I_{m+\Delta i, n+\Delta j}^{2}}}
$$

where $I_{m, n}$ represents the pixel intensity at the $(\mathrm{m}, \mathrm{n})$ location. An alternative is to build the correlation table from compressed format using the error correlation function [Hart 1996, Roth et al. 1995]. The error correlation function can be expressed as:

$$
\Phi_{\Delta i, \Delta j}^{e}=\frac{\sum_{m=1}^{M} \sum_{n=1}^{N}\left[I_{m, n}+I_{m+\Delta i, n+\Delta j}-\mid I_{m, n}-I_{m+\Delta i, n+\Delta j}\right]}{\sum_{m=1}^{M} \sum_{n=1}^{N}\left[I_{m, n}+I_{m+\Delta i, n+\Delta j}\right]}
$$

such that

$$
\Phi_{\Delta i, \Delta j}^{e}=1-\frac{\sum_{m=1}^{M} \sum_{n=1}^{N}\left[\left|I_{m, n}-I_{m+\Delta i, n+\Delta j}\right|\right]}{\sum_{m=1}^{M} \sum_{n=1}^{N}\left[I_{m, n}+I_{m+\Delta i, n+\Delta j}\right]}
$$

All three correlation methods are normalized such that a computed value of 1 indicates perfect correlation while a value of 0 indicates no correlation.

A schematic of a correlation window containing two exposures of seed particles is shown in Figure 1. It is clear from this figure that no polarity information can be obtained from a dualexposure PIV image; particles in the image could be moving to the left or to the right. Note that this correlation window generates three local maxima in the correlation table: the selfcorrelation peak, centered on the origin, and two conjugate displacement peaks on opposite sides of the origin, Figure 2.

Contrast this image with Figure 3, which shows a particle illuminated by three laser pulses separated by unequal time intervals. If the shorter time interval is assumed to occur between the first two laser pulses, then the particle in Figure 3(a) is traveling to the right while the particle in Figure 3(b) is traveling to the left. Autocorrelation of either figure, however, produces the same correlation table, Figure 4. This table contains seven peaks: the self- 
correlation peak plus two peaks for each pair of particle images selected from the three exposures.

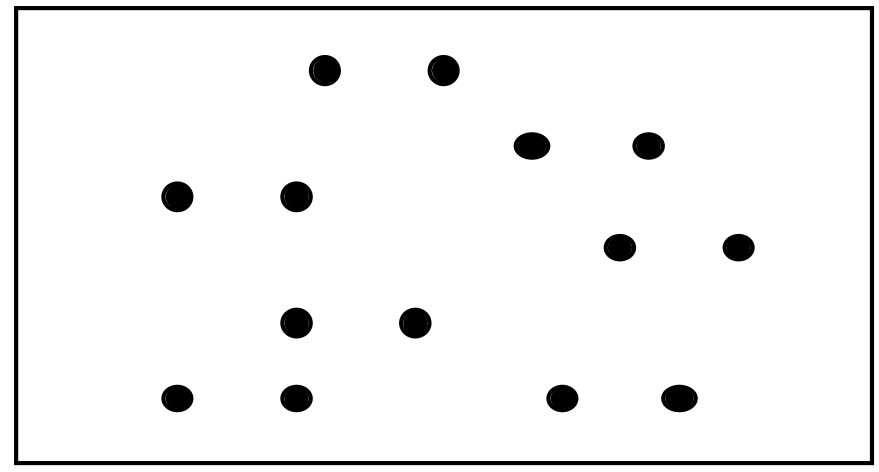

Figure 1 - Schematic of a correlation window within a double-exposed PIV image. The velocity in the window is directionally ambiguous.

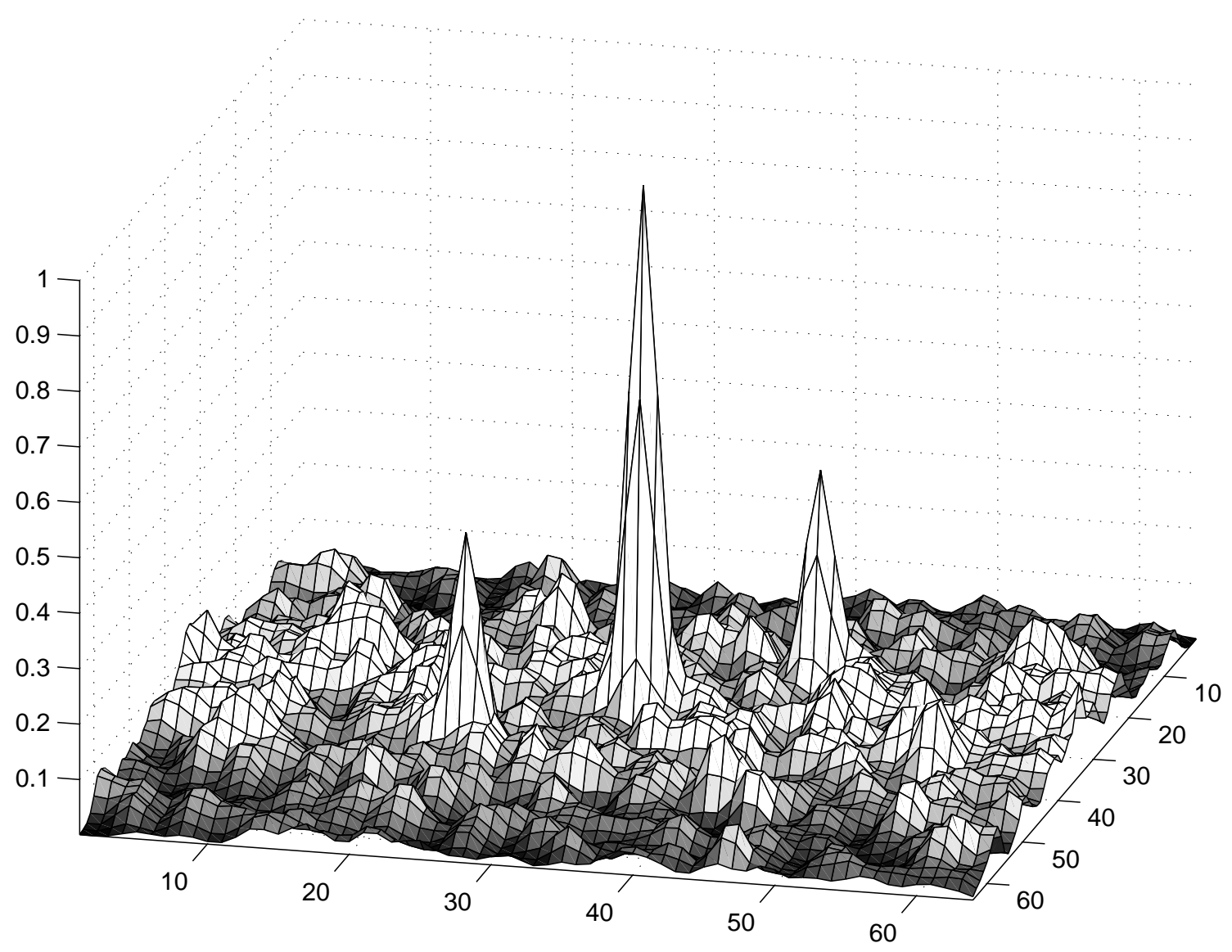

Figure 2 - Autocorrelation table produced by a double-exposed PIV image, showing three correlation peaks. The center, largest, peak represents the zero displacement correlation. 


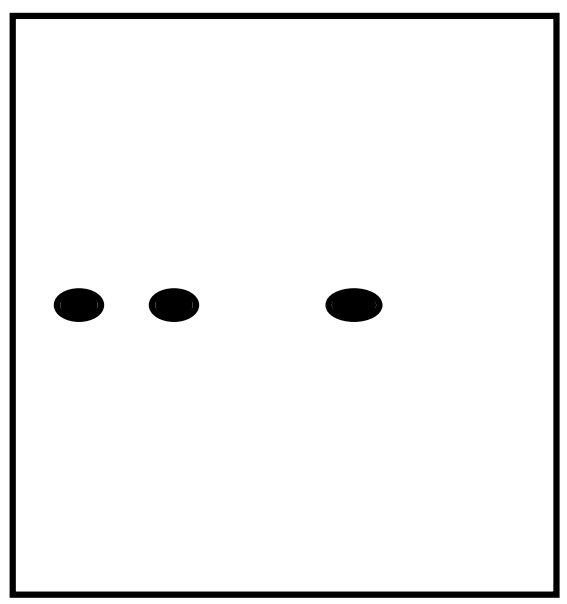

a)

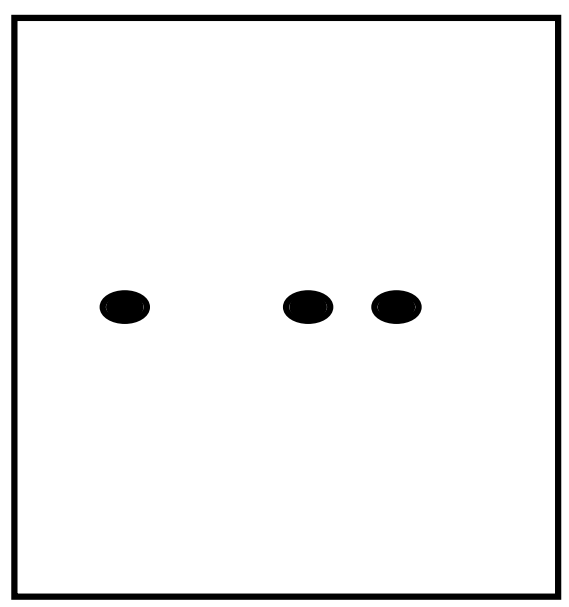

b)

Figure 3 - Schematic of a triple-exposed PIV image. Velocity in window a) is opposite to that in window b) for a given laser pulse sequence.

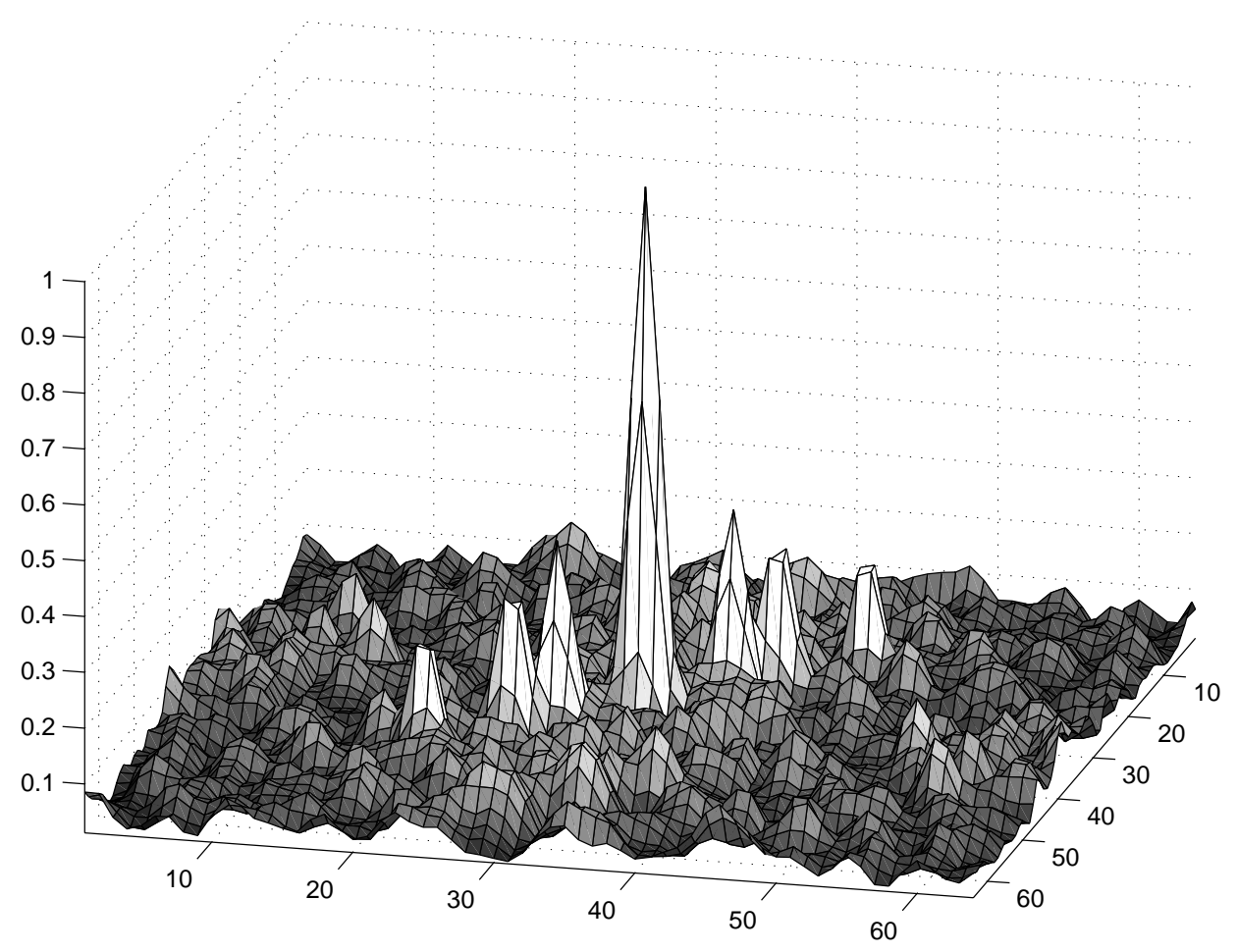

Figure 4 - Autocorrelation table produced by a triple-exposed PIV image, showing seven correlation peaks. The center, largest, peak represents the zero displacement correlation. The two sets of three smaller peaks represent the displacement between the first particle image and the second, the second and the third, and the first and the third. The images correlate both in the positive and the negative directions, generating two sets of three correlation peaks. 
Although the directionality of the flows indicated in Figures 3(a) and 3(b) can be readily discerned from the unequal spacing of the tracer particle images, the correlation tables computed from these images do not provide the information necessary to resolve this directionality. Thus, traditional PIV computational techniques cannot resolve the sign of the flow velocity although this information exists within the images themselves. Such ambiguity severely limits the usefulness of PIV autocorrelation for quantifying complex flows.

\section{ASYMMETRIC CORRELATION FUNCTION}

Directionality information that exists within triple exposed PIV images suggests the existence of an algorithm capable of resolving this information to within a finite probable error. Such an algorithm can be expressed as an asymmetric correlation function.

Consider the displacements indicated by the peaks in the correlation table computed in Figure 4. These displacements themselves do not provide sufficient information to resolve the sign of the flow velocity. The occurrences of these displacements, however, can themselves be correlated to yield a measure of directionality. If $\left(\Delta i_{1}, \Delta j_{1}\right),\left(\Delta i_{2}, \Delta j_{2}\right)$, and $\left(\Delta i_{3}, \Delta j_{3}\right)$ are the three primary displacements in Figure 3., with $\left|\Delta i_{1}\right|>\left|\Delta i_{2}\right|>\left|\Delta i_{3}\right|$, then the order in which the displacements occur relative to the coordinate origin of the image can be determined from the function,

$$
\begin{gathered}
\eta^{e}=\eta_{23}^{e}-\eta_{13}^{e}=\frac{\sum_{m=1}^{M} \sum_{n=1}^{N}\left|I_{m+\Delta i_{2}, n+\Delta j_{2}}-I_{m+\Delta i_{3}, n+\Delta j_{3}}+\right| I_{m, n}-I_{m+\Delta i_{3}, n+\Delta j_{3}}|-| I_{m, n}-I_{m+\Delta i_{2}, n+\Delta j_{2}}||}{\phi_{\Delta_{2}, \Delta i_{2}}^{e}+\phi_{\Delta_{3}, \Delta_{3}}^{e}} \\
-\frac{\sum_{m=1}^{M} \sum_{n=1}^{N}\left|I_{m+\Delta i_{1}, n+\Delta j_{1}}-I_{m+\Delta i_{3}, n+\Delta j_{3}}+\right| I_{m, n}-I_{m+\Delta \Delta_{3}, n+\Delta j_{3}}|-| I_{m, n}-I_{m+\Delta \Delta_{1}, n+\Delta \Delta_{1}}||}{\phi_{\Delta_{1}, \Delta_{1}}^{e}+\phi_{\Delta_{3}, \Delta_{3}}^{e}}
\end{gathered}
$$

where $\phi_{\Delta i_{x}, \Delta j_{x}}^{e}$ are the magnitudes of the displacement error correlation peaks before normalization, $I_{m, n}$ are pixel intensities, and the correlation window is again $\mathrm{M}$ by $\mathrm{N}$. The function $\eta_{13}$ is an error correlation of the displacements $\left(\Delta i_{1}, \Delta j_{1}\right)$ and $\left(\Delta i_{3}, \Delta j_{3}\right)$, while $\eta_{23}$ is an error correlation of $\left(\Delta i_{2}, \Delta j_{2}\right)$ and $\left(\Delta i_{3}, \Delta j_{3}\right)$. If $\left(\Delta i_{1}, \Delta j_{1}\right)$ occurs before $\left(\Delta i_{3}, \Delta j_{3}\right)$ relative to their assumed signs, then $\eta_{13}$ will be close to 1 and $\eta_{23}$ will be close to 0 , as in Figure 2(a). If a bright pixel is found $\left(\Delta i_{1}, \Delta j_{1}\right)$ units away from a reference pixel, then a bright pixel should be found $\left(\Delta i_{3}, \Delta j_{3}\right)$ units away from the same reference. The converse situation applies to Figure 3(b). Thus, if $\eta$ is greater than zero, then the first correlation peak occurs before the second. If $\eta$ is less than zero, then the second correlation peak occurs before the first relative to the direction in which the three correlation peaks are chosen (i.e., the assumed sign of the correlation.).

Note that $\eta$ has been defined in the same manner as the error correlation function. It can also be defined using a statistical correlation relationship; 
$\eta^{S}=\frac{\sum_{m} \sum_{n}\left(I_{m, n} \cdot I_{m+\Delta i_{1}, n+\Delta j_{1}}\right)\left(I_{m, n} \cdot I_{m+\Delta i_{i}, n+\Delta j_{3}}\right)}{\phi_{\Delta i_{1}, \Delta j_{1}}^{s} \cdot \phi_{\Delta i_{3}, \Delta j_{3}}^{s}}-\frac{\sum_{m} \sum_{n}\left(I_{m, n} \cdot I_{m+\Delta i_{2}, n+\Delta \dot{j}_{2}}\right)\left(I_{m, n} \cdot I_{m+\Delta i_{3}, n+\Delta j_{3}}\right)}{\phi_{\Delta i_{2}, \Delta j_{2}}^{s} \cdot \phi_{\Delta i_{3}, \Delta j_{3}}^{s}}$

where $\phi_{\Delta_{1}, \Delta_{1}}^{s}, \phi_{\Delta_{i_{2}, \Delta_{2}}}^{s}$ and, $\phi_{\Delta i_{3}, \Delta_{j}}^{s}$ are the magnitudes of the three primary non-zero displacement statistical correlation peaks before normalization. As long as the correlation peaks defined in the denominators of equations (3) or (4) indicate the true relative correlation, $\eta$ takes on either a positive or a negative value depending on the sign of the velocity relative to the assumed sign. A normalized asymmetric correlation function, however, is useful for determining the probability that a calculated sign is valid. The function $\eta$ can also be defined for spectral correlation. There is, however, no savings in computational intensity as $\eta$ requires only a single pass through an image to resolve directionality. The additional computations required to determine $\eta$ as defined either by equation (3) or (4) are minimal relative to those required to determine the correlation table.

\section{Simple Example}

To illustrate the use of the asymmetric autocorrelation function, consider the 10x10-pixel image shown in Figure 5. This image consists of three pixels with intensities of 1.0 at coordinates $(\Delta \boldsymbol{i}, \Delta \boldsymbol{j})=(3,3),(6,6)$ and $(8,8)$. The remaining pixels in the image have intensities of 0.0 :

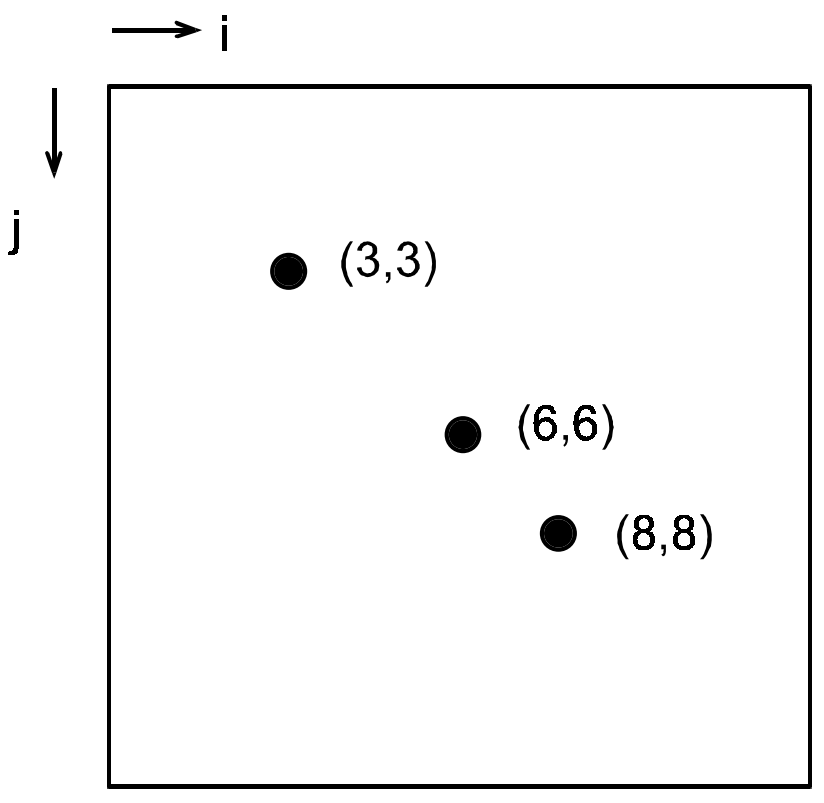

Figure 5 - Simple 10x10 pixel image containing three pixels with intensity equal to 1.0. This example is used to illustrate the asymmetric autocorrelation technique used to eliminate the $180^{\circ}$ ambiguity associated with autocorrelation.

An autocorrelation of this image using either the error correlation function or the statistical correlation function yields peak correlations at $\left(\Delta \boldsymbol{i}_{1}, \Delta \boldsymbol{j}_{1}\right)=(8-6,8-6)=(2,2), \quad\left(\Delta \boldsymbol{i}_{2}, \Delta \boldsymbol{j}_{2}\right)=(6-3,6-$ 
3) $=(3,3)$, and $\left(\Delta \boldsymbol{i}_{3}, \Delta \boldsymbol{j}_{3}\right)=(8-3,8-3)=(5,5)$ in the positive $\boldsymbol{i}, \boldsymbol{j}$ direction relative to the zero displacement correlation. From autocorrelation alone, it is impossible to tell how the exposures are ordered. We can, however, use the function $\eta$ defined in equation (3) or (4) to resolve this ambiguity. The non-normalized error correlation values $\phi_{\Delta i_{1}, \Delta_{1}}^{e} \phi_{\Delta i_{2}, \Delta_{2}}^{e}$ and, $\phi_{\Delta j_{3}, \Delta_{3}}^{e}$ for these peaks are all equal to $\phi^{\mathrm{e}}=1.0+1.0-|1.0-1.0|=2.0$, while the non-normalized statistical correlation values are equal to $\phi^{s}=1.0 * 1.0=1.0$. The asymmetric correlation, $\eta$, for this image is then equal to -1 using either definition of $\eta-$ equation 3 or equation 4 . Thus, the image correlates with a larger displacement first, $\left(\Delta \boldsymbol{i}_{2}, \Delta \boldsymbol{j}_{2}\right)=(3,3)$, followed by a smaller displacement, $\left(\Delta \boldsymbol{i}_{1}, \Delta \boldsymbol{j}_{1}\right)=(2,2)$, relative to the origin, or upper left corner, of the image.

\section{RESOLVING PARTICLE TRIOS}

Difficulties associated with asymmetric autocorrelation center on the resolution of peaks in the correlation table. Three collinear peaks, representing the three primary displacements in a correlation window, must be found for the asymmetric autocorrelation function to produce meaningful results. Statistically, this requirement poses a greater probability of error than does the single peak-finding of the autocorrelation of double-exposed PIV images.

Autocorrelation of triple-exposed PIV images is subject to the same limitations as the autocorrelation of double-exposed images. The density of tracer particles in a triple-exposed image, however, must be less than that in a corresponding double-exposed image, as there exist three, rather than two, images for any given particle. Although the same overall number of particle images may exist, the smaller number of tracer particles within a triple-exposed PIV image results in a reduction in the correlation signal strength. Consider that if two identical double-exposed PIV images are shifted relative to one another by the primary correlation peak displacement and compared, one particle from each of the two images aligns for every one that does not. This results in a reduction in magnitude of the primary correlation peak relative to the zero displacement correlation position, where all particle images are aligned. Triple-exposed PIV images introduce an additional uncorrelated particle when compared in this manner and thus have an additional 33\% reduction in the primary peak correlation values relative to the zero displacement correlation position. Knowledge of the pulse timing in a triple-exposed PIV image, however, allows most of the limitations associated with peak resolution to be circumvented. Only the smallest of the three primary displacements need be determined from the correlation table; if velocity gradients within the correlation window are not excessive, the other two primary displacements are scalar multiples of the first. The ratio of the three displacements mirrors the ratio of the times between exposures.

Limiting the search of the correlation table to a search for the smallest primary displacement allows implementation of an effective signal-strengthening technique. After the autocorrelation table of a subwindow has been calculated, each correlation value within the table can be replaced by the sum of its value plus the values at the locations where the second 
and third displacements would occur if the current location were the smallest of the tracer particle displacements. This position dependent mask can be expressed as,

$$
\Phi_{i, j}^{o}=\Phi_{i, j}+\Phi_{i+\tau(i-N / 2), j+\tau(j-M / 2)}+\Phi_{i+(1+\tau)(i-N / 2), j+(1+\tau)(j-M / 2)}
$$

where $\tau$ is the asymmetric exposure ratio (the ratio of the second exposure time interval to the first). In this manner, knowledge of the two larger displacement peaks is used to strengthen the smallest primary displacement peak. Once the smallest primary displacement is found, the other two primary displacements can be calculated from the asymmetric exposure ratio. Thus, asymmetrically-spaced particle trios can be used in much the same way multiple symmetrically-spaced exposures are used to improve correlation signal strength.

As an example of signal-strengthening using this position dependent mask technique, consider the $128 \times 128$ pixel image in Figure 6. Shown is a correlation subwindow taken from an experimental PIV image of a confined vortex, like that of Figure 11. The center of the correlation table corresponding to this window is shown in Figure 7, before position dependent masking, and in Figure 8, after position dependent masking. The smallest primary displacement peak at $\left(\Delta \boldsymbol{i}_{1}, \Delta \boldsymbol{j}_{1}\right)=(1,9)$, is clearly visible in Figure 8; other than the selfcorrelation, it is the largest local maximum. In Figure 7, however, this peak is obscured. Any search for the largest local maximum would overlook this peak and produce spurious results.

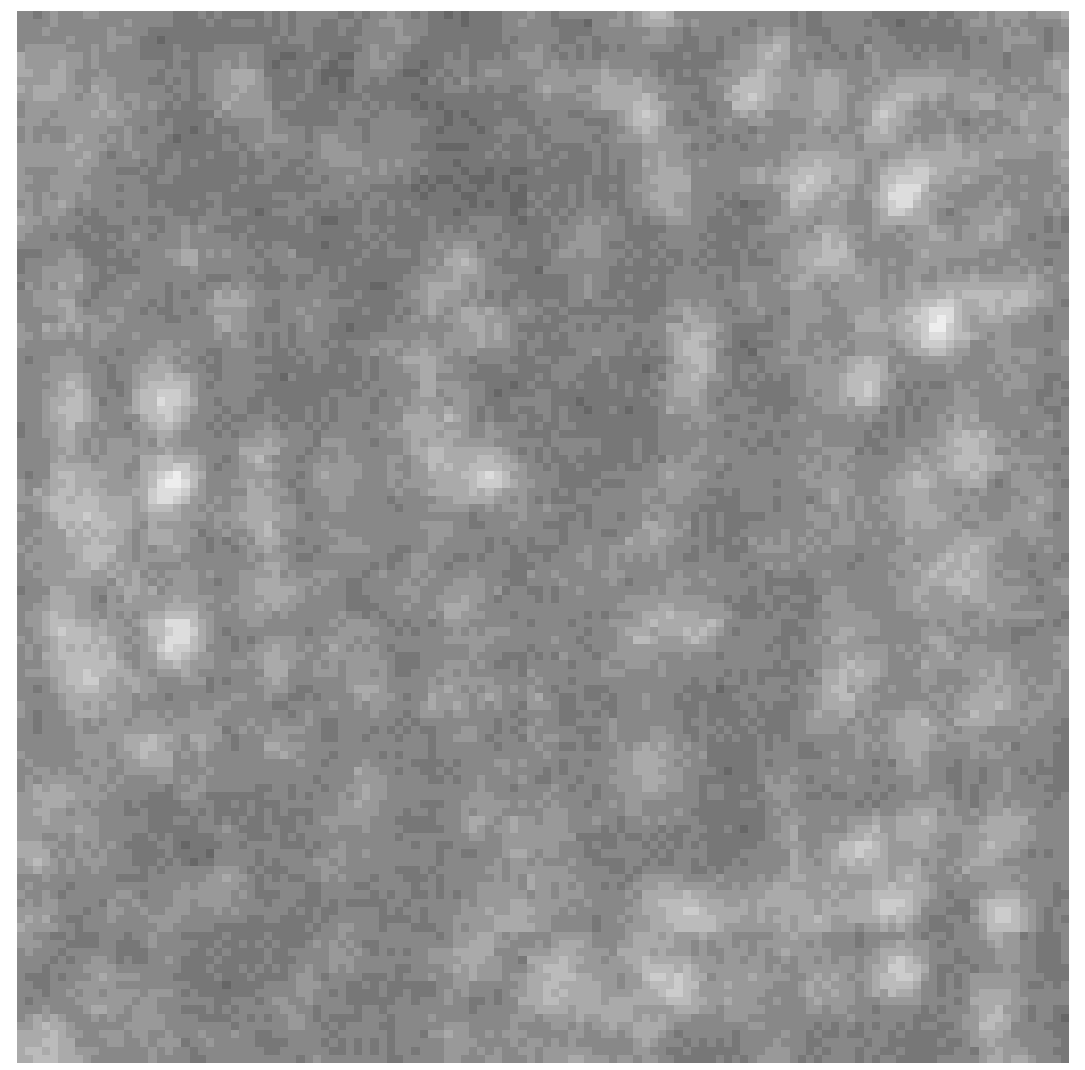

Figure 6 - Correlation window from an experimental PIV image of a turbulent vortex, containing three asymmetrically-spaced exposures. 


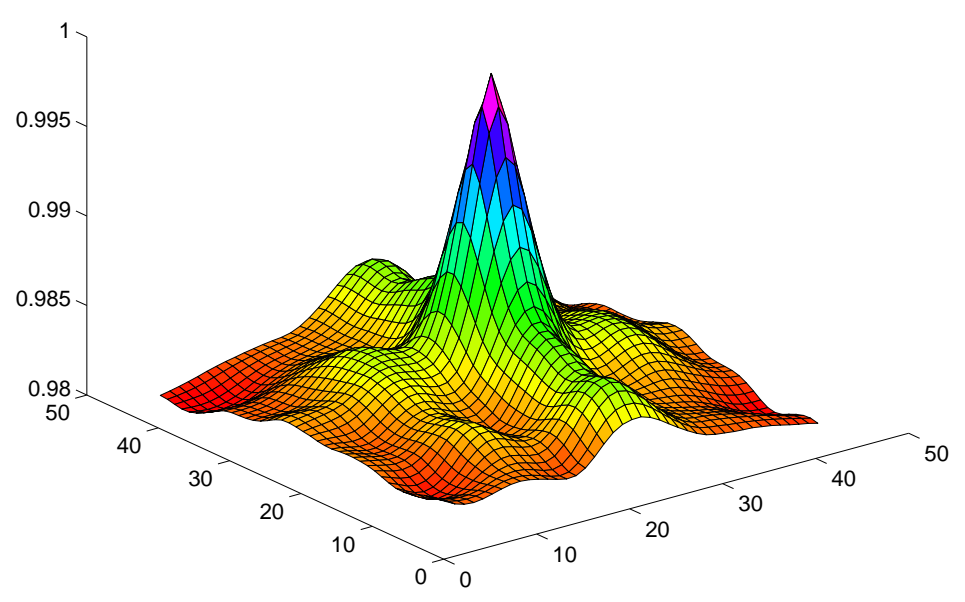

Figure 7 - Autocorrelation table from a triple-exposed experimental PIV image. The primary correlation peak representing the smallest displacement between particle images is obscured. The peak representing the second smallest displacement can be seen as a local maximum near the edge of the correlation table.

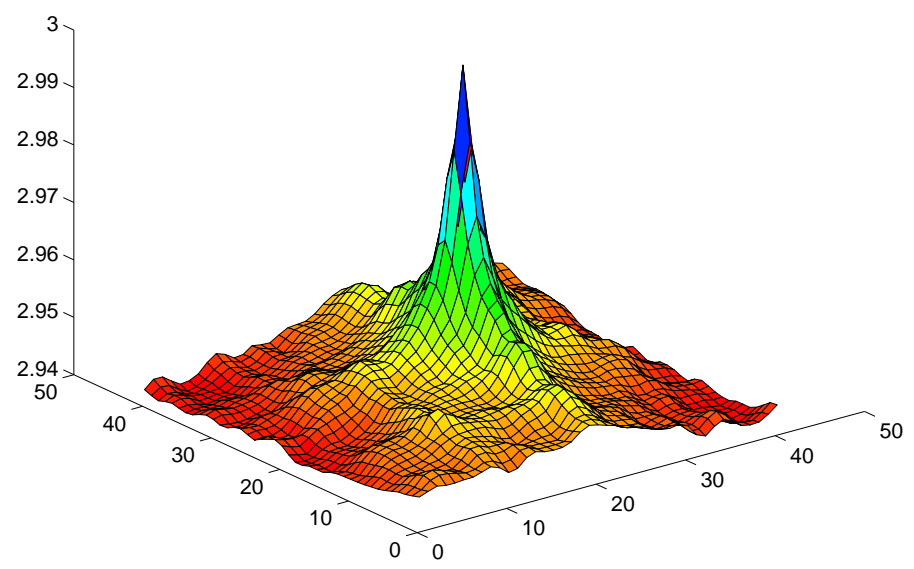

Figure 8 - Autocorrelation table from a triple-exposed experimental PIV image after the position dependent mask described by equation 5 has been applied. The primary correlation peak representing the smallest displacement between particle images is visible as a local maximum just below the zero correlation peak. 


\section{DEMONSTRATION}

\section{Synthetic Image}

The directionality function was tested on a synthetic three-pulse PIV image, shown in Figure 9. The 500x500-pixel image simulates the velocity field of a line vortex. Particle starting locations were chosen randomly, and displacements for the subsequent two exposures were calculated according to the velocity field. The time separating the first two exposures was taken to be half the time separating the second and third exposures. Each particle was given a radially symmetric Gaussian profile of intensity, approximately 5 pixels in diameter; these pixel intensities extended across the entire 8-bit grayscale spectrum. Approximately 2000 particle trios were placed in the image.

Figure 10 shows the vector map generated by error autocorrelation and evaluation of the directionality function. Spurious vectors are visible near the center of the vortex; these products of poor correlation arise because velocity gradients near the center are large relative to the 64x64-pixel correlation windows used to analyze the image-a common problem in PIV. Directional agreement with the ideal velocity field of the vortex, however, is good; less than $2 \%$ of the vectors have incorrect polarities. The occurrence of incorrect polarities points to the fact that the directionality function, $\eta$, will almost never take its ideal value of 1 or -1 ; incomplete particle trios within a correlation window, variable particle intensities, artifacts of the random placement of particles, and, in an experimental image, noise, ensure that $\eta$ will lie somewhat closer to the origin. The polarity criterion used for the vector map in Figure 10 was simply the sign of $\eta$. Values of $\eta$ that are particularly close to 0 , however, point to poor correlation, and cast uncertainty not only on the sign of the velocity vector but on the magnitude and direction of the vector computed in autocorrelation.

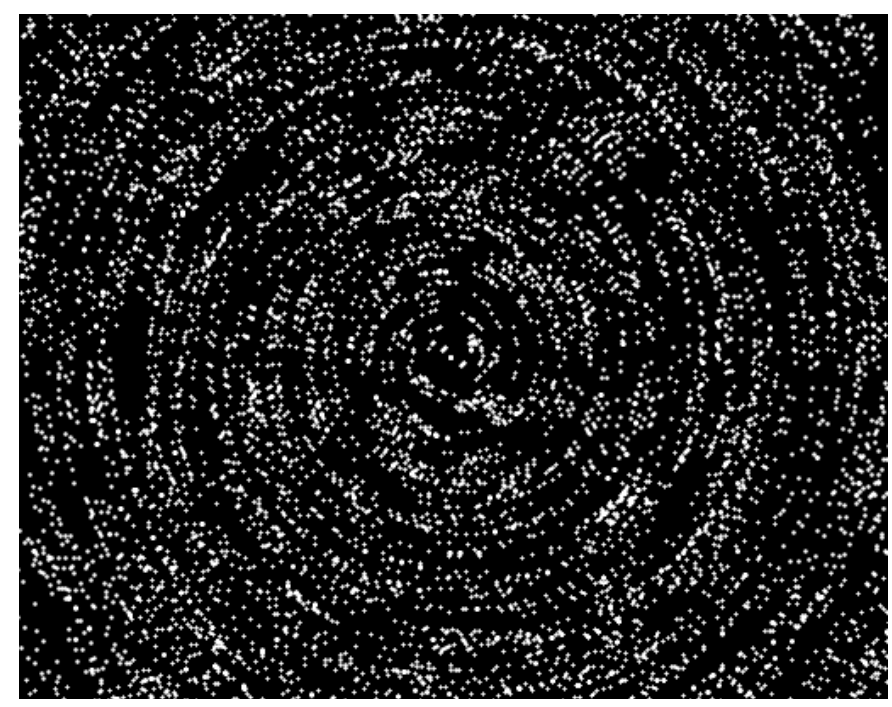

Figure 9 - Synthetic 500x500 pixel PIV image, simulating the velocity field of a line vortex. This image contains three asymmetrically-spaced exposures at an interval ratio, $\tau$, of two. 


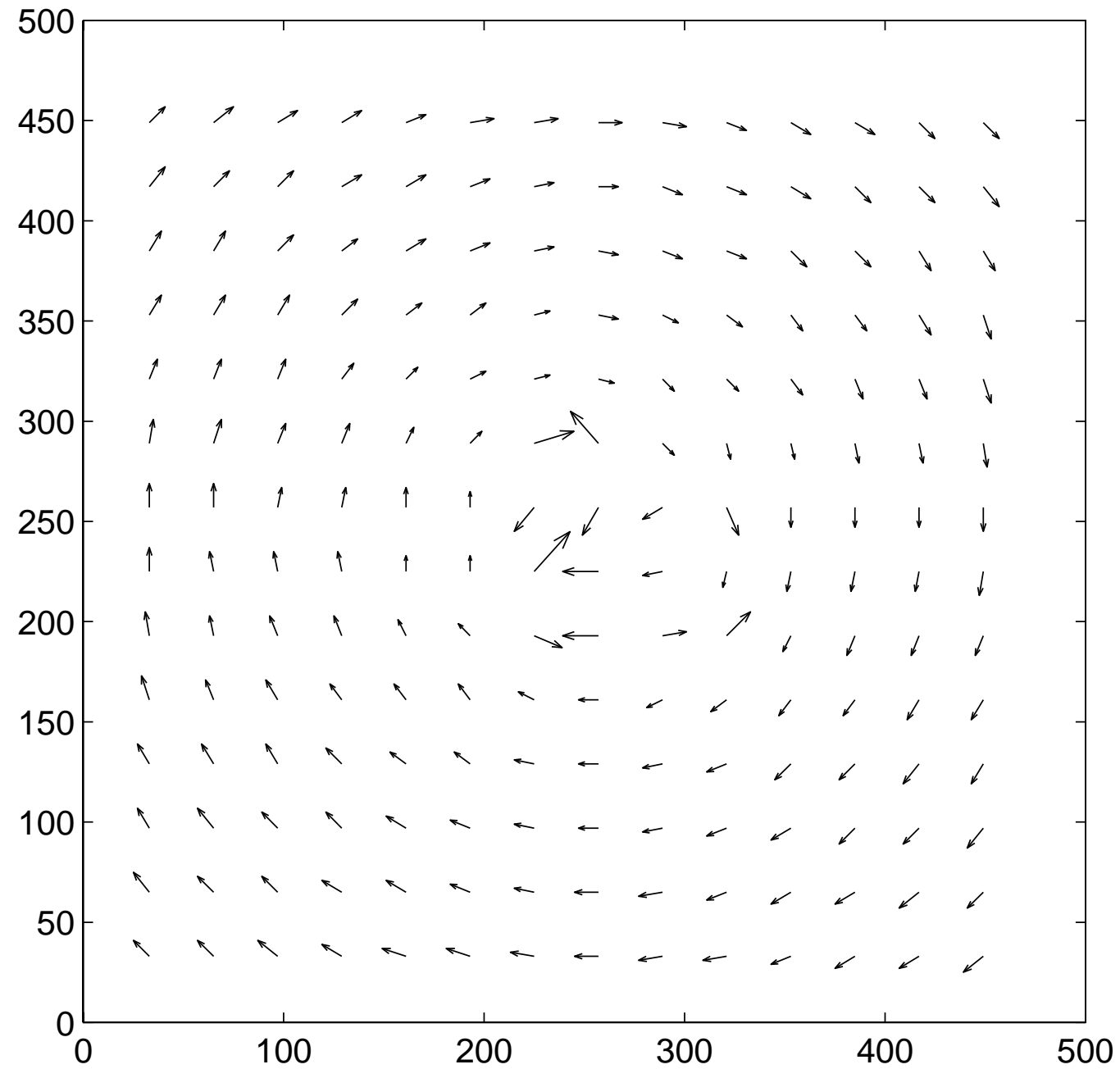

Figure 10 - Velocity field of the synthetic image, as determined by autocorrelation and evaluation of the asymmetric correlation function to determine directionality. Spurious vectors at the center of the image are due to velocity gradients that are large relative to correlation window size.

\section{Experimental Image}

The correlation algorithm and directionality function were also used to find the velocity field of an experimentally-obtained PIV image. An experimental image provides several potential sources of error not present in the synthetic image of the previous section. These include variations in particle brightness and focus, particles entering and exiting the plane of laser light between exposures, and background light noise.

The flow used to produce the PIV image was a turbulent vortex of water confined in a Plexiglas cylinder approximately $0.1 \mathrm{~m}$ in diameter. The swirl number of the vortex was 2.0, and tangential velocities were near $1 \mathrm{~m} / \mathrm{s}$. A pulsed Nd:YAG laser, frequency-doubled to produce visible light at $532 \mathrm{~nm}$, illuminated axial cross-sections of the flow. A Q-switch incorporated into the laser cavity permitted pulse separations as short as $20 \mu s$, but for PIV 
photographs of the vortex flow, the Q-switch was set to produce three pulses separated by 1.0 $m s$ and $2.0 \mathrm{~ms}$ intervals.

Polyester-based particles incorporating a fluorescent rhodamine dye were used to seed the flow. The diameters of these particles were on the order of $50 \mu \mathrm{m}$. Images were captured on Fuji Provia film with a 35-mm camera, and then digitized. An optical filter on the camera only passed light at the emission wavelength of the rhodamine dye, thus blocking out stray light from the laser and the room. This produced high-contrast PIV photographs, as seen in Figure 11.

Figure 12 shows a vector map of the turbulent vortex generated by autocorrelation and evaluation of the directionality functions, $\eta$. As with the synthetic image, velocity gradients near the center of the vortex are large relative to correlation window size, producing spurious vectors-a common situation in PIV. Spurious vectors near the outside edge of the plot are the result of particle images reflected in the glass wall of the experimental test section. Aside from these difficulties, asymmetric autocorrelation works extremely well; approximately $7 \%$ of the vectors in this example have incorrect sign.

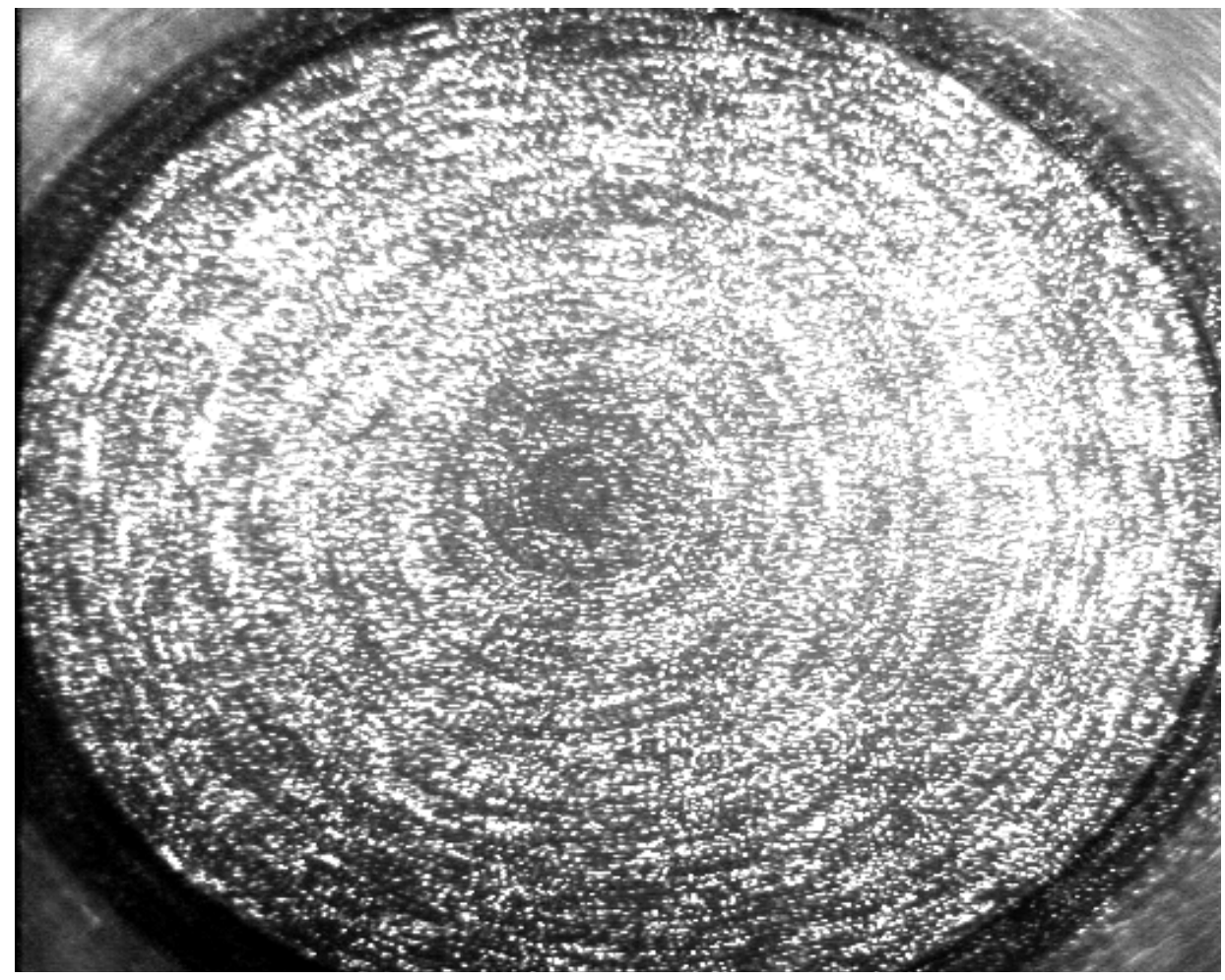

Figure 11 - Experimental PIV image of a turbulent vortex. Image contains three asymmetrically-spaced exposures. Particle reflections in the glass wall can be seen at the corners of this image. 


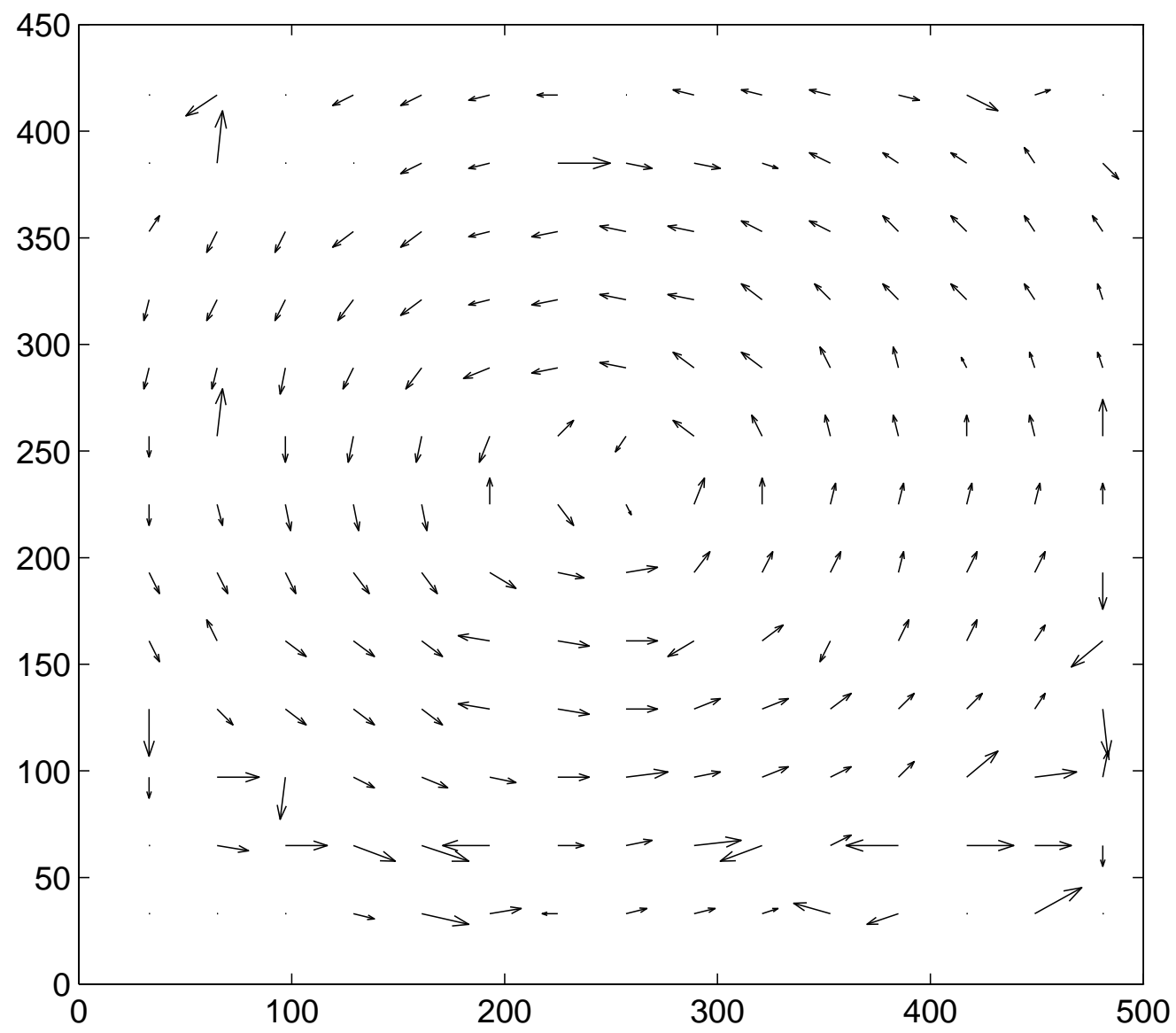

Figure 12 - Velocity field of the experimental image, as determined by autocorrelation and evaluation of the asymmetric correlation function to determine directionality. Spurious vectors along the outside edge are due to reflections of particles on the glass wall of the test section. Spurious vectors near the center of the velocity field are due to large velocity gradients within the correlation windows.

\section{CONCLUSIONS}

The asymmetric autocorrelation function provides a measure of the polarity of a vector found by autocorrelation. The velocity field of a flow can thus be calculated without directional ambiguity from a single triple-exposed PIV image. Demonstrations on a synthetic PIV image and on an experimentally obtained PIV image provide evidence of this function's accuracy.

Though asymmetric autocorrelation may introduce some limits on flow seeding and pulse timing, and can be hampered by strong velocity gradients or acceleration within the flow, simple signal-strengthening techniques that take advantage of the presence of three exposures in a PIV image vastly improve the reliability and accuracy of correlations. Asymmetric autocorrelation thus holds much promise in high-speed, high resolution PIV or HPIV applications where cross-correlation or image shifting between exposures in autocorrelation is impossible. It is particularly well suited to the study of high-speed flows containing complex vortex structures or strong turbulence. 


\section{REFERENCES}

Adrian, R.J. (1986): "Image Shifting Technique to Resolve Directional Ambiguity in Double-Pulsed Velocimetry." Appl Optics 25, 3855-3858.

Adrian, R.J. (1991): "Particle Imaging Techniques for Experimental Fluid Mechanics." Annual Review of Fluid Mechanics 23, 261-304.

Grant I.; Liu A. (1990): "Directional Ambiguity Resolution in Particle Image Velocimetry by Pulse Tagging." Exp Fluids 10, 71-76.

Hart, D.P. (1996): "Sparse Array Image Correlation.” 8th International Symposium on Applications of Laser Techniques to Fluid Mechanics, Lisbon, Portugal.

Landreth, C.C.; Adrian, R.J. (1988): "Electrooptical Image Shifting For Particle Image Velocimetry.” Appl Optics 27, 4216-4220.

Landreth, C.C.; Adrian, R.J.; Yao, C.S. (1988): "Double Pulsed Particle Image Velocimeter with Directional Resolution for Complex Flows." Exp Fluids 6, 119-128.

Lourenco, L.M. (1993): "Velocity Bias Technique for Particle Image Velocimetry Measurements of High-Speed Flows." Appl Optics 32, 2159-2162.

Oschwald M.; Bechle S.; Welke S. (1995): "Systematic Errors in PIV by Realizing Velocity Offsets With the Rotating Mirror Method.” Exp Fluids 18, 329-334.

Reeves M.; Garner, C.; Dent, J.C.; Halliwell, N.A. (1994): "Particle Image Velocimetry Measurements of Barrel Swirl in A Production Geometry Motored Optical i.c. Engine.” SAE Congress and Expo, paper 940281.

Reeves M.; Lawson N.J.; Halliwell N.A.; Coupland J.M. (1995): "Particle Image Velocimetry: Image Labeling Using Encoding Of The Point-Spread Function By Application Of A Polarization-Sensitive Pupil Mask.” Appl Optics 34, 194-200.

Roth, G.; Hart, D.; Katz, J. (1995): ’Feasibility of Using the L64720 Video Motion Estimation Processor (MEP) to increase Efficiency of Velocity Map Generation for Particle Image Velocimetry (PIV).” ASME/JSME Fluids Engineering and Laser Anemometry Conference, Hilton Head, South Carolina, 387-393.

Willert, C.E.; Gharib, M. (1991): “Digital Particle Image Velocimetry.” Exp Fluids 10, 181-193.

Zhang Z.; Eisele K. (1995): “The Two Dimensional Velocity Shift Caused By the Use of A Rotating Mirror in PIV Flow Field Measurements.” Exp Fluids 20, 106-111. 